\title{
Desfolhamentos contínuos e seqüenciais simulando danos de pragas sobre a cultivar de soja BRS $137^{1}$
}

\author{
Continuous and sequential defoliations to simulate pest injury in soybean \\ variety BRS 137
}

\author{
João Luiz Reichert ${ }^{2}$ Ervandil Corrêa Costa $^{3}$
}

\section{RESUMO}

O objetivo deste trabalho foi avaliar os efeitos dos desfolhamentos contínuo e seqüencial, tanto na fase vegetativa como reprodutiva, sobre o rendimento de grãos de soja (cultivar BRS 137), utilizando os níveis de danos estabelecidos para o manejo de pragas nessa cultura. O trabalho foi realizado na Universidade de Passo Fundo, RS, na safra 1999/2000. O delineamento experimental foi blocos ao acaso e os tratamentos, com quatro repetições, foram: desfolhamentos contínuos de (i) 33\% nas fases V4 a V9 e de (ii) 17 e (iii) 33\% nas fases R1 a R4 (escala de FEHR \& CAVINESS, 1977); desfolhamento seqüencial de (iv) $33+17 \%$ e (v) $17+33 \%$, respectivamente nas fases $V 4$ a V9 e nas fases $R 1$ a $R 4$; e (vi) testemunha (sem desfolhamento). Observou-se que os niveis de desfolhamento de $33 \%$ na fase vegetativa e $17 \%$ na reprodutiva, não afetaram o rendimento de grãos $\left(\mathrm{kg} \mathrm{ha}^{-1}\right)$, enquanto o desfolhamento seqüencial de $33+17 \%$ e $17+33 \%$ nas fases vegetativa e reprodutiva, respectivamente, e $33 \%$ na reprodutiva, reduziram o rendimento de grãos. $O$ rendimento de grãos por planta, número de legumes normais e de grãos, e o peso do grão foram os componentes determinantes na redução do rendimento de grãos de soja $\left(\mathrm{kg} \mathrm{ha}^{-1}\right)$, devido os desfolhamentos seqüenciais de $33+17 \%$ e $17+33 \%$. Todos os desfolhamentos reduziram o número de grãos por planta e de legumes normais por planta. Considerando os niveis de desfolhamento recomendados para o controle de insetos filófagos, pode ser indicado, para a cultivar BRS 137, desfolhamento de 33\% na fase vegetativa (V4 até V9) ou 17\% na fase reprodutiva ( $R 1$ até $R 4)$.

Palavras-chave: desfolhamento artificial, nível de ação, soja.

\begin{abstract}
The objective of this research was to evaluate the effect of both continuous and sequential defoliation on soybean yield (variety BRS 137). The treatments were evaluated during the vegetative and reproductive stages by using the injury levels recommended for the management of soybean defoliating pests. The study was conducted at the University of Passo Fundo, in Passo Fundo-RS, from December 1999 to May 2000. The experimental design was complete randomized blocks with six treatments and four replications. The following treatments were tested: (i) 33\% of continuous defoliation at stages V4 to V9 and of (ii) 17 and (iii) 33\% at stages R1 to R4 (FEHR \& CAVINES, 1977, stage); sequential defoliation of (iv) $33+17 \%$ and (v) $17+33 \%$ from stages $V 4$ to $V 9$ and from stages $R 1$ and R4; and (vi) control (no defoliation). The defoliation levels $33 \%$ in the vegetative stage and $17 \%$ in the reproductive stage did not affect grain yield $\left(\mathrm{kg} \mathrm{ha}^{-1}\right)$. In contrast, the sequential defoliating levels of $33+17 \%$ and $17+33 \%$ in the vegetative and reproductive stages, respectively, caused an yield reduction for BRS 137 soybean variety. The grain yield, the number of pods, number of grains, and the weight of grains were the most important components in determining the yield decrease with the sequential leaf defoliation of $33+17 \%$ and $17+33 \%$. All the leaf losses for decreased the number of grains/plant and the number of pods/plant. Considering the leaf action levels recommended to control of the leaf-feeding insects in soybean, it should be indicated for the BRS 137 soybean variety a leaf loss of $33 \%$ in the stages V4 to V9 or $17 \%$ in the stages R1 to $R 4$.
\end{abstract}

Key words: artificial defoliation, action level, soybean.

${ }^{1}$ Parte da Dissertação de Mestrado do primeiro autor.

${ }^{2}$ Engenheiro Agrônomo, MSc, Faculdade de Agronomia e Medicina Veterinária (FAMV), Universidade de Passo Fundo (UPF), 99001-970, Passo Fundo. E-mail: reichert@upf.tche.br Autor par correspondência.

${ }^{3}$ Engenheiro Agrônomo, Doutor, Professor Titular, Departamento Defesa Fitossanitária, Centro de Ciências Rurais (CCR), Universidade Federal de Santa Maria (UFSM), 97105-900, Santa Maria. 


\section{INTRODUÇÃO}

A importância da cultura da soja no Brasil pode ser constatada pela crescente expansão da área, pois, segundo BONATO \& BONATO (1987), no início da década de 70 eram cultivados no Brasil 1.318 .000 ha e, atualmente, de acordo com IBGE (2000), esta área atingiu 13.608.000 ha. Entre os diversos fatores que contribuíram para essa expansão da soja, destaca-se o aproveitamento de área, de máquinas e equipamentos e de mão-de-obra, quando cultivada em sucessão ao trigo, além das condições favoráveis de mercado, especialmente a exportação do produto (BONATO \& BONATO, 1987).

No Brasil, a maioria dos insetos fitófagos que se alimentam da soja são desfolhadores, e o dano que causam à soja são de grande importância econômica. Desse modo, visando determinar os períodos críticos do ataque desses insetos e estabelecer critérios para o uso de inseticidas, foram determinados os "níveis de ação" para o seu controle (TURNIPSEED, 1972; KOGAN et al., 1977; PANIZZI et al., 1977; MARQUES, 1978; PANIZZI, 1980).

Para o estabelecimento do nível de ação na soja, é desejável determinar o nível de desfolhamento necessário para reduzir o rendimento, assim como o estágio de desenvolvimento da planta, no qual o desfolhamento é mais crítico. Quando da avaliação do nível de ação, devem-se utilizar modelos de simulação artificial de desfolhamento e o rendimento como resposta da soja à desfolha (TODD \& MORGAN, 1972; TURNIPSEED, 1972).

Existem diversas referências sobre a capacidade da soja de, em certas fases de seu desenvolvimento, suportar determinados níveis de eliminação de folhas ou de parte delas sem que haja prejuízo no rendimento de grãos. O rendimento da soja é menos prejudicado por desfolhamentos na fase vegetativa do que na fase reprodutiva (TURNIPSEED, 1972; TEIGEN \& VORST, 1975; GAZZONI \& MINOR, 1979; SALVADORI \& CORSEUIL, 1979; GAZZONI \& MOSCARDI, 1998; HAILE et al., 1998 e RIBEIRO \& COSTA, 2000). O período mais crítico ao desfolhamento é o da formação ao enchimento de legumes (BEGUN \& EDEN, 1965; TURNIPSEED, 1972; GAZZONI, 1974; TEIGEN \& VORST, 1975; GAZZONI \& MINOR, 1979; SALVADORI \& CORSEUIL, 1979; GAZZONI \& MOSCARDI, 1998; HAILE et al., 1998; RIBEIRO \& COSTA, 2000).

Existem diversos estudos sobre tipos de desfolhamentos, desde os aplicados em apenas uma oportunidade, permanecendo, então, as plantas intactas até o final do ciclo (BEGUN \& EDEN, 1965;
ROSAS, 1967; GAZZONI, 1974; TEIGEN \& VORST, 1975; SALVADORI \& CORSEUIL, 1979; GAZZONI \& MOSCARDI, 1998); desfolhamento contínuo, no qual um dado grau de desfolhamento é mantido até determinada fase/estádio da planta, pela eliminação semanal das folhas novas ou parte delas; e desfolhamento seqüencial, no qual um nível de desfolhamento contínuo é realizado numa determinada fase/estádio de desenvolvimento da planta e outro nível de desfolhamento é realizada em outra fase/ estádio. Os desfolhamentos contínuos e/ou seqüenciais expressam mais fielmente a ação de insetos desfolhadores e aproximam-se da perda foliar e do estresse que a soja sofre quando infestações naturais de insetos ocorrem em diferentes estádios de desenvolvimento (TODD \& MORGAN, 1972; TURNIPSEED, 1972; GAZZONI \& MINOR, 1979; THOMAS et al., 1978; HAILE et al., 1998).

Os níveis de ação e os critérios para tomada de decisão no manejo de pragas da soja foram baseados, inicialmente, na porcentagem de desfolhamento em diferentes estágios da cultura e o nível populacional de lagartas (PANIZZI et al., 1977; MARQUES, 1978). A recomendação atual é que o controle com inseticidas deve ser feito com a presença de, em média, 20 lagartas grandes $(>1,5 \mathrm{~cm})$ por metro linear ou se o desfolhamento atingir 30\% antes do florescimento e 15\% tão logo apareçam as primeiras flores (REUNIÃO..., 1999). Essa recomendação não considera, ao adotar como critério o desfolhamento, se este é contínuo (30\% na fase vegetativa $+15 \%$ na reprodutiva) ou se os parâmetros de $30 \%$ e de $15 \%$ são independentes.

Este trabalho teve como objetivo, utilizando os níveis de ação preconizados pela pesquisa no manejo de pragas da soja, avaliar a resposta do cultivar BRS 137, submetido ao desfolhamento contínuo nas fases vegetativa ou reprodutiva, assim como o efeito do desfolhamento seqüencial nas duas fases.

\section{MATERIALEMÉTODOS}

O estudo foi desenvolvido em Passo Fundo, RS, na área experimental do Centro de Pesquisa Agropecuária (CEPAGRO) da Faculdade de Agronomia e Medicina Veterinária (FAMV) da Universidade de Passo Fundo (UPF), na safra 1999/ 2000. A semeadura da soja foi feita de acordo com as recomendações técnicas para a cultura no Rio Grande do Sul e Santa Catarina (REUNIÃO..., 1999). Empregouse uma semeadora adubadora de sete linhas para plantio direto, marca SHM Semeato ${ }^{\circledR}$, com espaçamento entre linhas de $45 \mathrm{~cm}$ e regulada com disco para semear vinte sementes aptas por metro, em solo classificado

Ciência Rural, v. 33, n. 1, jan-fev, 2003. 
como latossolo vermelho distrófico. Quando da semeadura da soja, a área estava com resteva da cultura anterior (cevada). A cultivar utilizada foi BRS 137 de ciclo semiprecoce. A adubação, e as demais práticas culturais, como controle de plantas daninhas, insetos, pragas e moléstias, foram realizadas de acordo com as recomendações para a cultura (REUNIÃO..., 1999). O delineamento experimental foi de blocos ao acaso, com quatro repetições, sendo que a unidade experimental constitui-se de cinco linhas de 2,5 m de comprimento. Foi considerada como área útil, para a colheita $\mathrm{e}$ obtenção dos dados, as três linhas centrais.

O experimento teve seis tratamentos, sendo cinco resultantes de diferentes níveis de desfolhamentos, sendo: desfolhamentos contínuos de (i) $33 \%$ na fase vegetativa, V4 até V9 e de (ii) $17 \%$ e (iii) $33 \%$ na fase reprodutiva, R1 até R4; desfolhamento seqüencial de (iv) $33+17 \%$ e (v) $17+33 \%$, em seqüência na fase vegetativa V4 até V9 e na fase reprodutiva R1 até R4; (escala de FEHR \& CAVINESS, 1977) e um tratamento (vi) testemunha, sem desfolhamento. Os desfolhamentos foram obtidos utilizando-se tesouras para a remoção de $1 / 2$ folíolo central (17\%) ou de todo o folíolo central (33\%). Semanalmente, as unidades experimentais foram repassadas para se fazer a manutenção do respectivo grau de desfolhamento, agindo-se em todas as folhas novas.

Para se obterem os dados, referentes aos componentes de rendimento, foram amostrados 20 plantas, colhidas ao acaso na área útil da parcela. A partir dessas plantas, foram separados, os legumes e debulhados manualmente, para determinação dos valores médios de rendimento/planta, números de legumes normais/planta, número de grãos/planta e peso de cem grãos. A colheita dos grãos foi realizada mecanicamente, e o rendimento da parcela foi transformado para $\mathrm{kg} \mathrm{ha}^{-1}$.

Para cada variável, foi realizado o teste de Lilliefors, para verificar a normalidade dos dados e, depois, a análise de variância (ANOVA), empregando o programa SAEG (FUNDAÇÃO ARTHUR BERNARDES, 1997), para determinar a significância do efeito dos níveis de desfolhamento e das fases de desenvolvimento da soja. $\mathrm{O}$ agrupamento das médias foi efetuado pelo teste de DUNCAN (1955), a 5\% de probabilidade de erro.

\section{RESULTADOS E DISCUSSÃO}

A análise de variância evidenciou efeitos significativos dos diferentes níveis de desfolhamento contínuos e seqüenciais sobre o rendimento de grãos da cultivar de soja BRS 137, os quais são apresentados na tabela 1 .

Não houve redução significativa no rendimento, quando o desfolhamento de $33 \%$ foi realizado apenas no estágio vegetativo, possivelmente devido a soja reagir ao desfolhamento, neste estágio, emitindo brotação contínua, caracterizando este comportamento da planta como uma tolerância ao ataque de insetos desfolhadores. Este resultado está de acordo com os obtidos por SALVADORI \& CORSEUIL (1979), CAVINESS \& THOMAS (1980), PICKLE\& CAVINESS (1984), GAZZONI\&MOSCARDI (1998) e RIBEIRO \& COSTA (2000), para os quais desfolhamentos no estágio vegetativo até o início da floração, não houve reduções significativas no rendimento da soja.

O desfolhamento de $17 \%$ na fase reprodutiva não reduziu significativamente o rendimento quando comparado a testemunha. Esta redução foliar, segundo TURNIPSEED (1972), é compensada com uma maior penetração da luz até as folhas inferiores, aumentando deste modo a produção de fotossintatos, e mantendo um rendimento de grãos semelhante a testemunha. Estes resultados concordam com os resultados obtidos por ROSAS (1967), SALVADORI \& CORSEUIL (1979), e RIBEIRO \& COSTA (2000) mas diferiram dos verificados por GAZZONI \& MINOR (1979) para desfolhamento com duração de dez dias. Uma redução acentuada no rendimento $(21,2 \%)$ foi ocasionada pela desfolha de $33 \%$ no estágio reprodutivo, diferindo significativamente da testemunha. Este período é o mais crítico na formação de legumes e enchimento de grãos, pois a redução da área foliar tem efeitos direto na e número de legumes normais de soja, submetida a reduções foliares contínuas e seqüenciais em diferentes estádios de desenvolvimento. Passo Fundo - RS, 2000 .

\begin{tabular}{|c|c|c|c|c|c|c|c|}
\hline \multicolumn{3}{|c|}{ Tratamentos } & \multirow{2}{*}{$\begin{array}{l}\text { Rendimento } \\
\left(\mathrm{kg} \mathrm{ha}^{-1}\right)\end{array}$} & \multirow{2}{*}{$\begin{array}{c}\text { Rendimento } \\
\text { planta (g) }\end{array}$} & \multirow{2}{*}{$\begin{array}{c}\text { No grãos }^{\mathrm{o}} \text { planta }\end{array}$} & \multirow{2}{*}{$\begin{array}{c}\text { Peso } \\
100 \\
\text { grãos }(\mathrm{g})\end{array}$} & \multirow{2}{*}{$\begin{array}{l}\mathrm{N}^{\mathrm{O}} \\
\text { legumes } \\
\text { normais }\end{array}$} \\
\hline $\mathbf{V}^{1}$ & & & & & & & \\
\hline $33 \%$ & + & $0 \%$ & $2.811 \mathrm{ab}^{3}$ & $10,01 \mathrm{ab}$ & $48,08 \mathrm{~b}$ & $20,72 \mathrm{a}$ & $23,84 \mathrm{~b}$ \\
\hline $33 \%$ & + & $17 \%$ & $2.688 \mathrm{~b}$ & $8,79 \mathrm{~b}$ & $46,25 \mathrm{~b}$ & $19,48 \mathrm{~b}$ & $22,18 b$ \\
\hline $17 \%$ & + & $33 \%$ & $2.755 \mathrm{~b}$ & $8,55 \mathrm{~b}$ & $44,62 \mathrm{~b}$ & $19,23 \mathrm{~b}$ & $21,81 \mathrm{~b}$ \\
\hline $0 \%$ & + & $17 \%$ & $2.862 \mathrm{ab}$ & $10,29 \mathrm{ab}$ & $49,02 \mathrm{~b}$ & $20,28 \mathrm{ab}$ & $23,98 \mathrm{~b}$ \\
\hline $0 \%$ & + & $33 \%$ & $2.624 \mathrm{~b}$ & $9,55 \mathrm{~b}$ & $48,06 \mathrm{~b}$ & $19,98 \mathrm{ab}$ & $23,62 \mathrm{~b}$ \\
\hline \multicolumn{3}{|c|}{ Testemunha } & $3.330 \mathrm{a}$ & $11,88 \mathrm{a}$ & $59,56 \mathrm{a}$ & $20,37 \mathrm{a}$ & $28,89 \mathrm{a}$ \\
\hline \multicolumn{3}{|c|}{ C. V. $(\%)$} & 8,41 & 13,64 & 12,40 & 2,53 & 11,55 \\
\hline
\end{tabular}

$1-\mathrm{V}=$ fase vegetativa;

2- $\mathrm{R}=$ fase reprodutiva;

3-As médias não seguidas pela mesma letra, diferem entre si, pelo teste de Duncan em nível a $5 \%$ de probabilidade de erro.

Ciência Rural, v. 33, n. 1, jan-fev, 2003. 
produção de fotossintatos e no acúmulo de matéria seca. Esse resultado concorda com os obtidos por TODD \& MORGAN (1972), em desfolhamento contínuo e nível de desfolhamento semelhante. De um modo geral, a redução obtida, é semelhante às observadas por BEGUN \& EDEN (1965), TURNIPSEED (1972), GAZZONI (1974), SALVADORI \& CORSEUIL (1979), e HAILE et al. (1998), que consideram serem as fases de formação e enchimento dos legumes as mais críticas ao rendimento. Os resultados obtidos neste estudo discordam dos observados por ROSAS (1967), assim como dos obtidos por GAZZONI \& MINOR (1979), com desfolhamento durante 10 dias. Outros resultados não coincidentes, são os de GAZZONI \& MOSCARDI (1998) e RIBEIRO \& COSTA (2000), assim como os de CAVINESS \& THOMAS (1980) e PICKLE \& CAVINESS (1984), com 25 e 50\% de redução da área foliar.

Observaram-se reduções significativas no rendimento quando houve remoção foliar seqüencial, combinando os níveis de desfolha de $33+17 \%$ e $17+33 \%$, nas fases vegetativa e reprodutiva, respectivamente. O desfolhamento seqüencial nos estágios vegetativos e reprodutivos da cultivar BRS 137, provocou reduções estatisticamente significativas de $642 \mathrm{~kg} \mathrm{ha}^{-1}$ com $33 \%$ na fase vegetativa $+17 \%$ na fase reprodutivà, e de 575 $\mathrm{kg} \mathrm{ha}^{-1}$, quando realizado com os percentuais inversos $(17+33 \%)$. Isso equivale a perdas de $19,2 \%$ e de $17,2 \%$ no rendimento da soja, respectivamente. Combinação semelhante, realizada por TURNIPSEED (1972), não diferiu significativamente da testemunha, quanto ao rendimento.

O comportamento do cultivar BRS 137, frente aos diferentes níveis de desfolhamento e em diferentes estádios fenológicos, indica que, quando do uso do desfolhamento como critério para o manejo de pragas, deve-se considerar $33 \%$ no período vegetativo ou $17 \%$ no período reprodutivo para o controle de desfolhadores, pois a adição dos dois níveis $(33+17 \%)$ de desfolhamento acarretou reduções significativas na produção de grãos. A análise de variância mostrou efeito significativo dos tratamentos quanto ao rendimento por planta. Os resultados obtidos (Tabela 1) com 33\% de desfolhamento no período vegetativo não diferiram significativamente do demais tratamentos. Esta redução foliar não diferiu da testemunha, concordando com os resultados de GAZZONI (1974), GAZZONI \& MINOR (1979), SALVADORI \& CORSEUIL (1979) e GAZZONI \& MOSCARDI (1998), mas diferem dos dados de ROSAS (1967). Resultado semelhante foi obtido com $17 \%$ de desfolhamento no período reprodutivo, divergindo dos obtidos por ROSAS (1967) e SALVADORI \& CORSEUIL (1979).
O rendimento das plantas não foi reduzido pelos desfolhamentos de $33 \%$ na fase vegetativa e de $17 \%$ na reprodutiva. Verificou-se que os desfolhamentos combinados de $33+17 \%$, e de $17+33 \%$ nos períodos vegetativo e reprodutivo, respectivamente, proporcionaram uma diminuição significativa de rendimento em relação à testemunha. Estes dois níveis de redução foliar nos diferentes estádios, diminuem a produção de fotossintatos e nutrientes, afetando significativamente o rendimento por planta. Constatou-se efeito significativo do desfolhamento de $33 \%$ no período reprodutivo quanto ao rendimento das plantas. Os resultados foram semelhantes aos obtidos por ROSAS (1967), GAZZONI (1974) e SALVADORI \& CORSEUIL (1979), mas divergentes dos observados por RAMIRO \& OLIVEIRA (1975), GAZZONI \& MINOR (1979) e GAZZONI \& MOSCARDI (1998). A resposta do rendimento por planta ao desfolhamento, dentro de cada fase de desenvolvimento, foi semelhante à resposta da variável rendimento/hectare. Esses resultados concordam com os obtidos por GAZZONI (1974) e SALVADORI \& CORSEUIL (1979).Para o número de grãos por planta, a análise de variância evidenciou efeito significativo dos tratamentos sobre essa variável. Na Tabela 1, verifica-se que os efeitos dos desfolhamentos não diferiram entre si, porém, reduziram significativamente essa variável, independentemente do grau e da fase de desenvolvimento da cultura em que foram aplicados, em relação à testemunha. Esses dados discordam dos obtidos por SALVADORI \& CORSEUIL (1979) e RIBEIRO \& COSTA (2000) quanto ao nível de $17 \%$ de desfolhamento, mas concordam inteiramente com os dados obtidos com $33 \%$ pelo último autor e, em parte, com os do primeiro autor. Os desfolhamentos seqüenciais de $33+17 \%$ e $17+33 \%$ e de $33 \%$ no estágio reprodutivo reduziram significativamente o número de grãos. Esta redução refletiu diretamente no rendimento por planta e, conseqüentemente, por área.

Os diferentes níveis de desfolhamentos contínuos e seqüenciais, apresentaram efeito significativo sobre o peso de 100 grãos, conforme pode ser observado na análise de variância. Os resultados obtidos (Tabela 1) evidenciaram que desfolhamentos realizados somente no período vegetativo $(33 \%)$ ou reprodutivo (17 e 33\%) não reduziram significativamente o peso de 100 grãos, em relação à testemunha. Resultados idênticos foram observados, com os mesmos níveis de desfolhamentos, por ROSAS (1967), GAZZONI \& MINOR (1979), SALVADORI \& CORSEUIL (1979) e RIBEIRO \& COSTA (2000) e, com nível menor de desfolha, por TURNIPSEED (1972). 
O efeito de $33 \%$ de desfolhamento realizado durante a fase vegetativa, sobre o peso de 100 grãos, está em concordância com os estudos de TODD \& MORGAN (1972), GAZZONI (1974), RAMIRO \& OLIVEIRA (1975) e GAZZONI \& MOSCARDI (1998). Já esse mesmo nível de redução foliar no estágio reprodutivo não reduziu o peso do grão, concordando com GAZZONI \& MOSCARDI (1998), mas divergindo dos dados obtidos com desfolhamentos contínuos de TODD \& MORGAN(1972) e THOMAS et al. (1978), assim como nos desfolhamento simples de TURNIPSEED (1972) e GAZZONI (1974), que observaram reduções significativas no peso do grão. Estes níveis de desfolhamento, independente do estádio, não reduziram a produção de fotossintatos e, conseqüentemente não interferiram na formação e enchimento dos grãos. Verificou-se uma significativa diminuição do peso do grão, em relação à testemunha, quando desfolhamentos contínuos foram aplicados nos estádios vegetativos e reprodutivos, independente da combinação dos níveis percentuais. Segundo GAZZONI \& MINOR (1979), entre os vários componentes do rendimento importantes na redução do rendimento, destaca-se o peso do grão.

O número de legumes normais por planta, de acordo com a análise de variância foi significativamente afetado pelos níveis de desfolhamentos. Na Tabela 1, verifica-se que não houve diferença estatística, para essa variável, entre os desfolhamentos. Porém, ocorreu uma diminuição significativa no número de legumes nos tratamentos com desfolhamento em relação à testemunha, na qual houve produção mais elevada de legumes normais por planta. Redução significativa no número de legumes normais/planta no desfolhamento com $33 \%$ na fase vegetativa, em relação à testemunha, também foram observada por ROSAS (1967) e RAMIRO \& OLIVEIRA (1975). Mas, estes resultados diferem dos resultados de GAZZONI (1974), TEIGEN \& VORST (1975), GAZZONI \& MINOR (1979), SALVADORI \& CORSEUIL (1979), GAZZONI \& MOSCARDI (1998) e RIBEIRO \& COSTA (2000).

As reduções significativas no número de legumes normais por planta, observada para os desfolhamentos de 17 e 33\% no estágio reprodutivo, são provocadas pela redução foliar e conseqüente perda da área fotossintética no período mais crítico da soja, que é o período do início da formação e enchimento dos legumes. Esses resultados estão de acordo com os resultados obtidos por ROSAS (1967) e SALVADORI \& CORSEUIL (1979), mas discordam dos observados por GAZZONI \& MINOR (1979) e RIBEIRO \& COSTA (2000). O mesmo foi verificado com o nível de $33 \%$ de desfolhamento por GAZZONI (1974) e GAZZONI \& MOSCARDI (1998).

Os desfolhamentos contínuos e seqüenciais de $33+17 \%$ e de $17+33 \%$, nas fases vegetativa e reprodutiva, resultaram em uma redução no número de legumes normais por planta e, de acordo com GAZZONI \& MOSCARDI (1998), esse componente do rendimento é o mais afetado por desfolhamentos elevados e o principal responsável pela redução do rendimento $\left(\mathrm{kg} \mathrm{ha}^{-1}\right)$. Esses dados permitiram verificar que a cultivar BRS 137 apresentou uma redução elevada de legumes normais por planta, independente do nível de desfolhamento e do estádio fenológico da planta.

\section{CONCLUSÕES}

Para as condições em que o experimento foi conduzido, os resultados permitem as seguintes conclusões para a cultivar BRS 137:

Os desfolhamentos seqüenciais de $33+17 \%$ e de $17+33 \%$ nas fases vegetativa e reprodutiva, e o desfolhamento de $33 \%$ na reprodutiva reduzem o rendimento de grãos $\left(\mathrm{kg} \mathrm{ha}^{-1}\right)$. O desfolhamento de $33 \%$ na fase vegetativa e $17 \%$ na reprodutiva, não afeta o rendimento de grãos de soja $\left(\mathrm{kg} \mathrm{ha}^{-1}\right)$;

Os componentes de rendimento determinantes na redução do rendimento de grãos de soja $\left(\mathrm{kg} \mathrm{ha}^{-1}\right)$, devido aos desfolhamentos seqüenciais de $33+17$ e $17+33 \%$, são o rendimento de grãos por planta, número de legumes normais, número de grãos, e o peso de grãos.

\section{REFERÊNCIASBIBLIOGRÁFICAS}

BEGUN, A., EDEN, W.G. Influence of defoliation on yield and quality of soybeans. Journal of Economic Entomology, Baltimore, v.53, n.3, p.591-592. 1965.

BONATO, E.R., BONATO, A.L.V. A soja no Brasil: história e estatística. Londrina : EMBRAPA- CNPSo, 1987. 61p. (Documentos, 21.)

CAVINESS, C.E., THOMAS, J.D. Yield reduction from defoliation of irrigated and non-irrigated soybeans. Agronomy Journal, Madison, v.72, n.6, p.977-980, 1980.

FEHR, W. R., CAVINESS, C. E. Stage of soybeans development. Ames : Iowa State University, 1977. 12p. (Special Report, 80.)

FUNDAÇÃO ARTHUR BERNARDES. SAEG. Versão 7.1, Sistema operacional MS-DOS e manual de codificação. Viçosa : 1997. CD.

GAZZONI, D.L. Avaliação de efeito de três níveis de desfolhamento aplicados em quatro estádios de crescimento de dois cultivares de soja (Glycine max (L.) MERRILL), sobre a produção e a qualidade do grão. 1974. 70f. Dissertação (Mestrado em Fitotecnia) - Faculdade de Agronomia, Universidade Federal do Rio Grande do Sul. 
GAZZONI, D.L., MINOR, H.C. Efeito do desfolhamento artificial em soja, sobre o rendimento e seus componentes. In: SEMINÁRIO NACIONAL DE PESQUISA DE SOJA, 1, 1978. Londrina, PR. Anais... Londrina : EMBRAPA/CNPS, 1979. v.2, p.47-57.

GAZZONI, D.L., MOSCARDI, F. Effect of defoliation levels on recovery of leaf area, on yield and agronomic traits of soybeans. Pesquisa Agropecuária Brasileira. Brasília, v.33 n.4, p.411-424, 1998.

HAile, F.J., Higley, L.G., SPECHT, J.E. Soybean cultivars and insect defoliation: yield loss and economic injury levels. Agronomy Journal, Madison. v.90, n.1, p.344-352, 1998.

IBGE. Prognóstico para 2001. Levantamento sistemático da produção agrícola. Rio de Janeiro, 2000. v.12 supl., p.114.

KOGAN, M. et al. Pilot insect pest management program for soybean in southern Brazil. Journal of Economic Entomology, v.70, n. 5, p.659-663, 1977.

MARQUES, G.L. Manejo de pragas na cultura da soja. Passo Fundo : EMBRAPA/CNPT, 1978. 29p. (Circular Técnica, 2).

PANIZZI, A.R. Manejo integrado de pragas da soja: situação atual e perspectivas futuras. In: CONGRESSO BRASILEIRO DE ENTOMOLOGIA, 6., 1980, Campinas, SP. Anais... Campinas : Fundação Cargill, 1980. p.215-244.

PANIZZI, A.R. et al. Insetos da soja no Brasil. Londrina : EMBRAPA-CNPSo, 1977. 20 p. (Boletim Técnico, 1).

PICKLE, C.S.; CAVINESS, C.E. Yield reduction from defoliation and planta cutoff of determinate and semideteminate soybean. Agronomy Journal, Madison. v.76, n.3, p.474476, 1984.
RAMIRO, Z. A.; OLIVEIRA, D. de A. Influência da desfolhação artificial na produtividade da cultura da soja. O Biológico, São Paulo, v.41, n.4, p.97-104, 1975.

REUNIÃO DE PESQUISA DA SOJA NA REGIÃO SUL, 27, 1999, Chapecó. Recomendações técnicas para a cultura da soja no Rio Grande do Sul e em Santa Catarina 1998/ 99.Chapecó, SC : EPAGRI, 1998. 133p

RIBEIRO, A. L. de P.; COSTA, E.C. Desfolhamento em estádios de desenvolvimento da soja, cultivar BR 16 , no rendimento de grãos. Ciência Rural, Santa Maria, v.30, n.5, p.767771,2000

ROSAS, G. S. Influencia de la defoliacion parcial em plantas de soya (Glycine max). Turrialba, Turrialba, v.17, n.1, p.193197, 1967.

SALVADORI, J.R., CORSEUIL, E. Efeitos de quatro níveis de desfolhamento aplicados em quatro estágios de desenvolvimento da soja (Glycine $\max$ (L.) Merril), na produção de grãos. Agronomia Sulriograndense, Porto Alegre, v.15, n.1, p.91-101, 1979.

TEIGEN, J. B.; VORST, J. J. Soybean response to stand reduction and defoliation. Agronony Journal, Madison, v.67, n.6, p.813-815, 1975.

THOMAS, G.D. et al. Effects of single and sequential defoliations on yield and quality of soybeans. Journal of Economic Entomology, Maryland, v.71, n.6, p.871-874, 1978.

TODD, J. W.; MORGAN, L. W. Effects of hand defoliation on yield and seed weight of soybeans. Journal of Economic Entomology, Maryland, v.65, n.2, p.567-570, 1972.

TURNIPSEED, S.G. Response of soybeans to foliage losses in South Carolina. Journal of Economic Entomology, Maryland, v.65, n.1, p.224-229, 1972 